\title{
Standardized Uptake Value
}

National Cancer Institute

\section{Source}

National Cancer Institute. Standardized Uptake Value. NCI Thesaurus. Code C69310.

An effectively dimensionless measure of regional tracer uptake calculated as the activity

concentration within a 2D region of interest ( $\mathrm{ROI}$ ) or 3D volume of interest (VOI)

measured on a PET image (nCi1 mL) / [injected dose (nCi) / body weight (g)]. 